\section{Le portail de l'Association canadienne de gastroentérologie, lancé en septembre 2009}

\author{
Alaa Rostom MD FRCPC M. Sc., président, comité \\ d'éducation de l'ACG, université de Calgary, Calgary, \\ Alberta
}

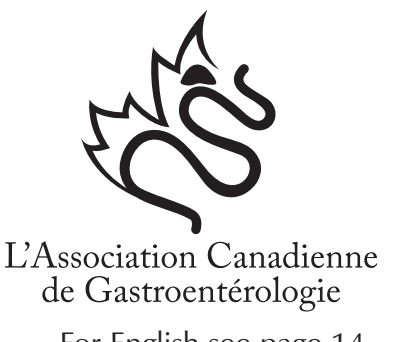

For English see page 14
Rienvenue dans le nouveau portail Bde formation (le portail) de l'Association canadienne de gastroentérologie (ACG), un espace Web consacré à aider les membres à se tenir à jour et à répondre à leurs besoins de formation et de maintien du certificat.

Le portail est accessible, en anglais, par le site Web de l'ACG, à www.cagacg.org. C'est un nouvel outil pour les membres, qui s'ajoute aux possibilités de formation pendant la Semaine canadienne des maladies digestives (SCMD) annuelle déjà offerte aux membres de l'ACG. Le portail vous permet de réviser des thèmes précis, d'effectuer de courtes autoévaluations, de discuter de sujets avec des collègues du pays entier, de produire des idées de projets d'apprentissage général, de s'informer des événements de l'ACG et plus encore. Le portail sera régulièrement enrichi de nouveau matériel d'apprentissage, compte tenu de vos commentaires.

Inscrivez-vous et explorez le portail. Jetez un coup d'œil à des conférences très bien cotées de la SCMD prononcées lors des congrès passés. Vérifiez vos connaissances avant et après avoir visualisé du matériel de formation. Testez votre savoir en endoscopie. Discutez de sujets d'actualité avec des experts et des collègues, tels que le dépistage du cancer du côlon avec le docteur Des Leddin.

Peut-être par-dessus tout, le portail retracera vos activités personnelles de formation et le temps que vous aurez passé dans le site Web. Vous pourrez réviser et imprimer votre sommaire d'activités où que vous soyez, en tout temps. Nous aimerions un jour pouvoir transmettre directement l'information au Collège royal des médecins et chirurgiens du Canada (CRMCC) et vous faire ainsi gagner du temps et vous éviter de transcrire les activités dans votre compte du CRMCC.

Amusez-vous, apprenez beaucoup et faites-nous parvenir vos commentaires pour que nous puissions nous améliorer.

QU'EST-CE QUE LE PORTAIL ? Le portail de l'ACG est le guichet vers les possibilités virtuelles de perfectionnement professionnel continu des membres de l'ACG. Les membres peuvent y accéder tout à loisir par l'entremise du site Web, à l'adresse www.cag-acg.org. Il faut un code d'ouverture de session et un mot de passe pour l'utiliser (le même que pour ouvrir une session dans la zone réservée aux membres du site Web de l'ACG). Si vous ne vous souvenez pas de votre code, vous pouvez communiquer avec le bureau de l'ACG ou cliquer sur Forgot your password? dans la zone réservée aux membres du site Web de l'ACG. Le système permettra aux membres de procéder à des évaluations personnelles des besoins et de se créer un profil personnel pour cibler des domaines d'intérêt.

Qu'y a-t-il dans le portail ?

Le portail contient divers programmes d'apprentissage et de formation, tels que les suivants :

suite à la page 17

\begin{tabular}{|lcc|}
\hline \multicolumn{3}{|c|}{ L'ACG est fière de présenter ses bienfaiteurs : } \\
$\begin{array}{l}\text { Abbott Canada } \\
\text { Olympus Canada Inc }\end{array}$ & AstraZeneca Canada Inc & Axcan Pharma Inc \\
& Pentax Canada Inc & Schering-Plough Canada Inc \\
& UCB Pharma Inc \\
\hline
\end{tabular}


- Conférences vidéo enrichies d'une vérification des connaissances avant et après la vidéo

- Conférences vidéo

- Forums de discussion

- Questionnaire sur l'endoscopie

- Ressources (p. ex., guides de pratique clinique, documents de principes)

Pourquoi utiliser le portail ?

- Centraliser vos activités et vos dossiers de formation.

- Utiliser la vaste gamme d'outils qu'offre le portail de l'ACG.

- Accumuler des crédits pour respecter les besoins d'apprentissage pour le maintien du certificat et profiter de l'inscription automatique de ces crédits dans votre profil personnel du portail.
- Voir les conférences auxquelles vous n'avez pas pu assister lors de congrès passés.

- Participer à des forums de discussion interactifs pour discuter avec des experts.

- Créer et effectuer facilement des programmes d'autoévaluation.

- Accéder à des ressources essentielles telles que des guides de pratique clinique et des documents de principes.

Revenez souvent dans le site www.cag-acg.org pour demeurer au courant de l'évolution de cette possibilité et accéder nombreux autres avantages offerts aux membres! 


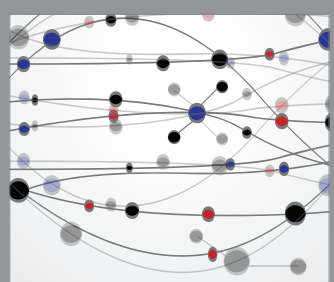

The Scientific World Journal
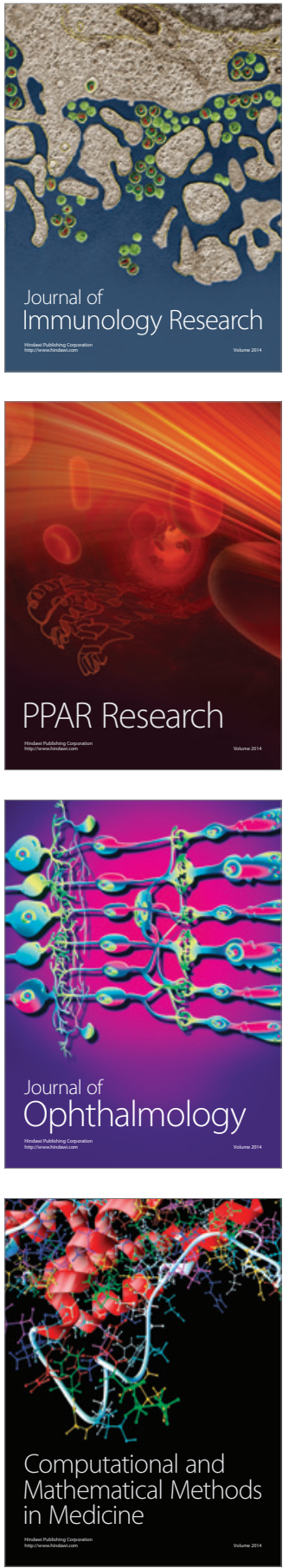

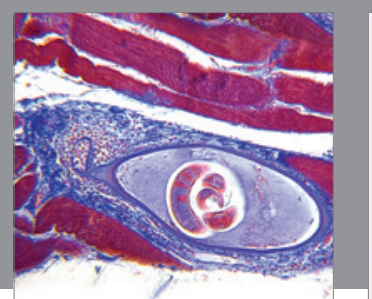

Gastroenterology Research and Practice

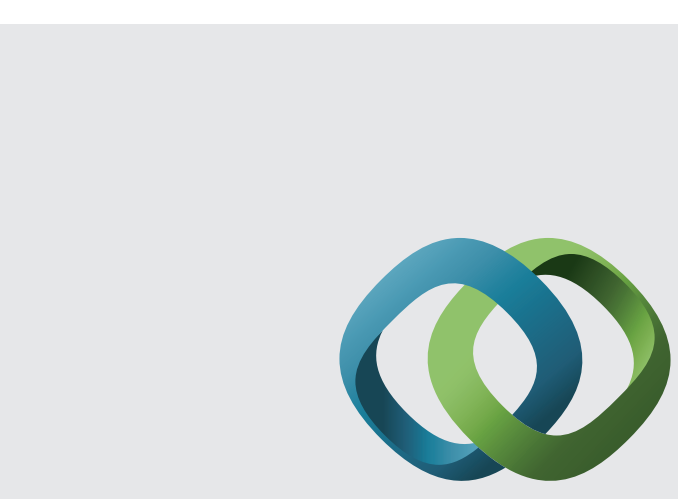

\section{Hindawi}

Submit your manuscripts at

http://www.hindawi.com
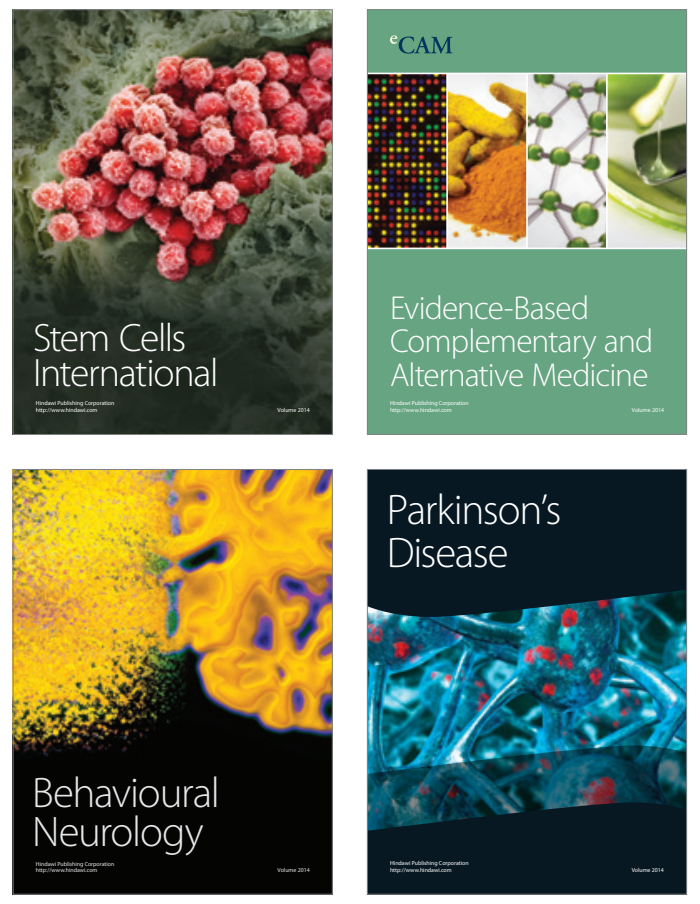
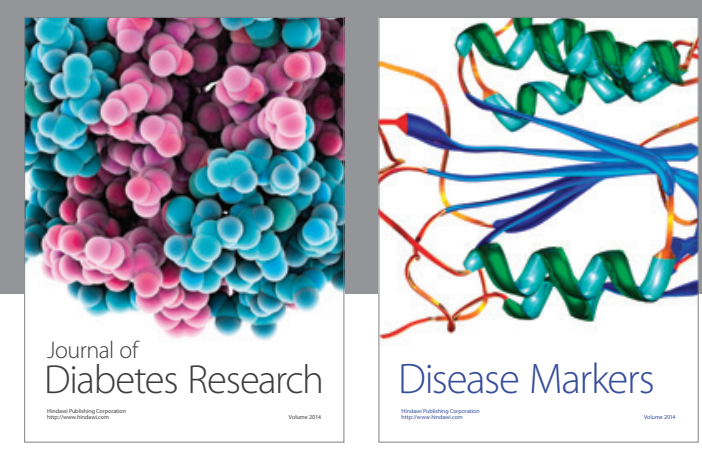

Disease Markers
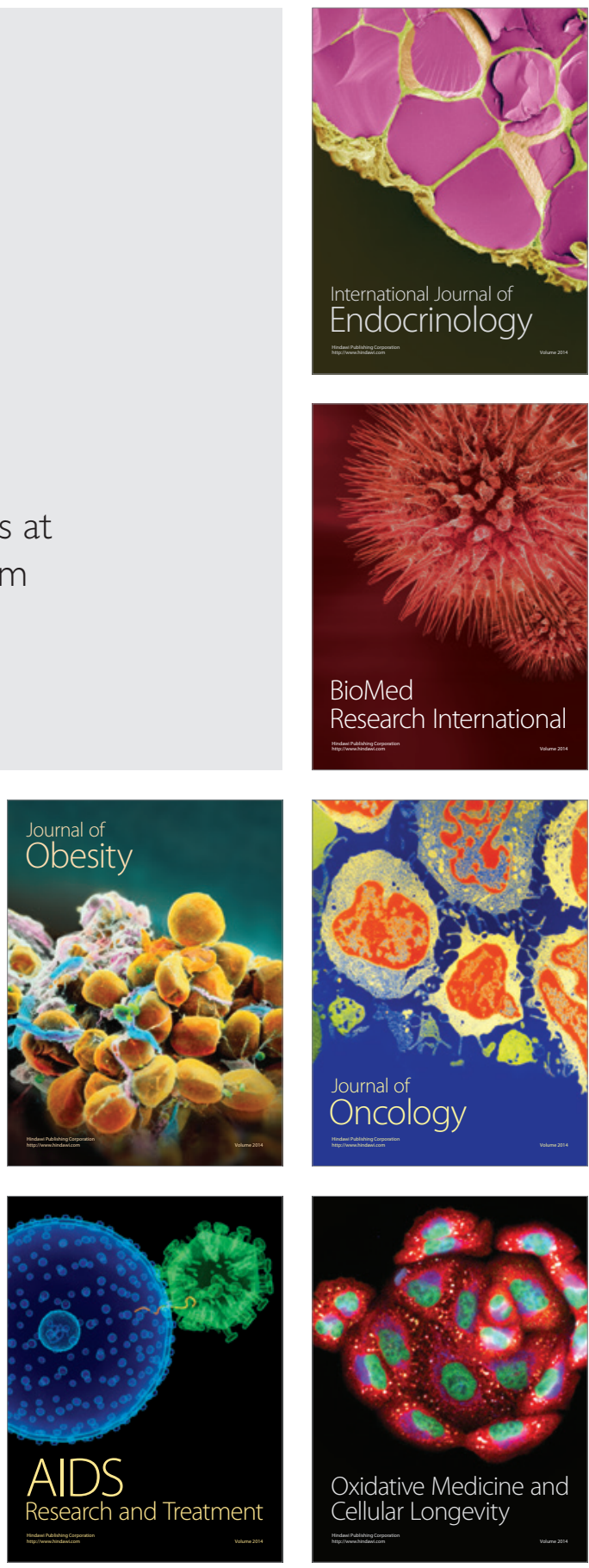